\title{
Formación de clases y conflicto social en el sistema previsional chileno: mecanismos de acumulación capitalista y desafíos para la democracia
}

\author{
Felipe Stefano Ruiz Bruzzone \\ Universidad de Chile \\ felipe.ruiz@ug.uchile.cl \\ https://orcid.org/0000-0001-5122-8775
}

\author{
How to cite this paper: \\ Ruiz Bruzzone, Felipe Stefano (2020). Formación de clases \\ y conflicto social en el sistema previsional chileno: \\ mecanismos de acumulación capitalista y desafíos para \\ la democracia. Revista Encuentros, Universidad Autónoma del \\ Caribe. Vol. 18-3. Dossier. \\ Doi: 10.15665/encuent.v18i3.2134
}

Recibido: 27 de noviembre de 2019 / Aceptado: 5 de enero de 2020

\section{RESUMEN}

El presente artículo se enfoca en una específica y poco estudiada dinámica de conformación de clases sociales en Chile, a saber, aquel proceso ocurrido al alero del sistema previsional privatizado. Para ello se analiza el papel que los fondos de pensiones privatizados han jugado en la acumulación capitalista en Chile, elemento que se relaciona con la creciente hegemonía que cobran las finanzas en los cursos de acumulación capitalista de la economía global. A partir de ello se propone una caracterización de las Administradoras de Fondos de Pensiones (AFP) como una fracción de la clase empresarial de Chile, retomando aportes del pensamiento social de orientación marxista. Se concluye ofreciendo una mirada panorámica sobre los desafíos que tales dinámicas han impreso sobre el carácter y sentidos de la democracia en Chile y América Latina.

Palabras clave: Capitalismo Financiero; Administradoras de Fondos de Pensiones; Chile Clases Sociales; Democracia.

\section{Class Formation and Social conflict in the Chilean Pension System: Mechanisms of Capitalist Accumulation and Challenges for Democracy}

\begin{abstract}
The present article focuses on a specific and less studied social class formation process in Chile, namely, that occurred at the eaves of the privatized pension system. For this purpose, the role that privatized pension funds have played in capitalist accumulation in Chile is analysed, which is related to the growing hegemony that finances hold in the courses of capitalist accumulation, globally. Based on this, the Chilean Pension Fund Administrators are characterized as a fraction of the business class in Chile, considering contributions from Marxist-oriented social thought. It concludes by offering a panoramic view of the challenges that such dynamics have imprinted on the character and meanings of democracy in Chile and Latin America.
\end{abstract}

Keywords: Financial capitalism - Pension Fund Administrators - Chile - Social Classes - Democracy 


\section{INTRODUCCIÓN}

\section{Presentación: las finanzas y el capitalis- mo (neoliberal) contemporáneo}

En diferentes sentidos el pensamiento social y económico haido señalando la importancia de las finanzas como componente explicativo de la estructuración contemporánea del capitalismo (Chesnais, 2017; Fumagalli \& Lucarelli, 2011; Lapavitsas, 2016). Las crisis recientes de la economía global ${ }^{66}$ y el papel determinante que en ellas han jugado las finanzas tornan necesario escudriñar en la evolución histórica de estos novedosos actores, en su comportamiento actual y en sus capacidades de determinación respecto a la totalidad social, política y económica.

Sin embargo, ante tal empresa las herramientas teóricas y analíticas del pensamiento social contemporáneo parecen insuficientes. Como plantea Esteban Torres (2017) para el caso latinoamericano, ello tiene que ver con dos abandonos por parte de la teoría y pensamiento social a la salida del siglo XX: por una parte, el abandono de las nociones de totalidad social, poder y conflicto social, como categorías estructurantes de cualquier tentativa de pensamiento crítico; la contracara de esta situación es el abandono explícito del esfuerzo por desarrollar - o actualizar - una teoría contemporánea del capitalismo, como marco de referencia obligado al momento de contextualizar los fenómenos sociales en estudio.

El presente artículo busca aportar - a partir de un estudio reciente y de carácter empírico - con una perspectiva de investigación que contribuya a superar los dos dilemas señalados. Se presentan los resultados de un proceso de investigación que se propuso dilucidar un proceso específico de conformación de clases sociales al alero del sistema previsional chileno (19802018), en específico, la emergencia de novedosos actores empresariales en su seno. Para ello en el segundo apartado se rescatan elementos de análisis que permiten situar la especificidad de las finanzas en el capitalismo contemporáneo, dando cuenta del papel específico que en tal sentido han jugado los fondos de pensiones en la economía chilena. Posteriormente, se

66 Específicamente la crisis financiera del período 2007-2008. propone una mirada analítica sobre el proceso reciente de conformación de clases sociales al alero del sistema previsional chileno, dando cuenta de la emergencia y consolidación de una novedosa fracción empresarial que parece volverse hegemónica en el direccionamiento de los cursos de acumulación y circulación de capital. Se concluye ofreciendo una mirada panorámica sobre la conflictividad social reciente en torno a la reproducción social en Chile y situando los desafíos que tales dinámicas han impreso sobre el carácter y sentidos de la democracia en tal país.

\section{Neoliberalismo y financiarización de las economías: el papel de la privatización de los fondos de pensiones en Chile}

\section{Las tendencias de la financiarización en el capitalismo contemporáneo}

Quizá uno de los antecedentes estructurales más potentes para comprender el sentido histórico del "giro neoliberal" sea aquel de la tendencia a la baja de la tasa de ganancia que se produjo de manera persistente desde 1965 en adelante tanto en las economías centrales de mayor relevancia de Norteamérica y Europa (Duménil \& Lévy, 2001), como en América Latina (O’Donnell, 1977). Esta situación ha sido explicada según un doble énfasis: por una parte se sostiene que el proceso de acumulación basado en la intensificación del trabajo alcanzó límites desde una dimensión propiamente técnica al finalizar la primera mitad del siglo XX: se alcanzaron límites objetivos en el desarrollo de las fuerzas productivas (Lapavitsas, 2009a); por otra parte, se ha observado que la contracara fue una tendencia al aumento de la conflictividad social a escala mundial a fines de la década de 1960, elemento indicativo de un límite en el marco de las relaciones sociales de producción vigentes a la fecha (Escalante, 2016; O’Donnell, 1977).

Ambos aspectos integran los componentes de la tenaza que limitó el despliegue del capitalismo durante gran parte del siglo XX - sobre todo en su ciclo 'keynesiano' luego de la crisis global de 1929-1930 (Escalante, 2016) - y resultan ser los antecedentes históricos de la posterior transformación neoliberal de los marcos institucionales de regulación de la economía y la política (Chesnais, 2003; Escalante, 2016). En este marco de 
transformaciones se asiste entonces a tres características emergentes en los procesos de acumulación de capital.

En primer lugar, la emergencia de actores sui generis del capitalismo financiarizado que se constituyen en una importante fuerza directiva de los procesos de acumulación de capital. Para Costas Lapavitsas (2009a, 2013b) la financiarización de las economías capitalistas representa la expansión de los mecanismos de acumulación sobre la esfera de la circulación, ante un progresivo estancamiento de la esfera productiva como fuente de ganancias. En este proceso se reconfiguran las relaciones entre los principales actores de la economía (empresas industriales, comerciales, trabajadores e instituciones financieras) y emergen otros que pueden denominarse mediante el término finanzas: bancos, fondos de inversión, sociedades financieras en sentido estricto como también departamentos financieros en empresas no definidas como financieras (Chesnais, 2017).

En segundo lugar, la consolidación de las finanzas como eje directivo de los procesos acumulación capitalista contemporáneos hace que se configure una lógica de producción de ganancias que supone su creciente influencia en las esferas de la circulación del capital y de la reproducción, estimulando una racionalidad económica basada en el rentismo y la expropiación financiera. ${ }^{67}$ El movimiento histórico hacia una circulación ampliada del capital se puede captar al observar la reciente emergencia histórica de diversos mecanismos de producción de ganancias en los procesos de intermediación de activos financieros (tasa de interés, comisiones de intermediación, etc.) que permiten captar una parte del plusvalor ya producido en las relaciones estrictamente de producción, para re inyectarlo a los circuitos de valorización (Chesnais, 2003; Lapavitsas, 2009b).

La principal consecuencia de estas transformaciones es que tal tipo de estrategias han ido penetrando en la racionalidad de los diversos actores económicos: para el caso de las empresas, durante las tres últimas décadas éstas se han ido involucrando de manera creciente en procesos de intermediación financiera (Lapavitsas, 2009a). En el caso de la clase trabajadora, se ha producido un doble movimiento de financarización: por una parte

67 Este término será explicado en los siguientes párrafos. se ha introducido la deuda en los hogares como forma de resolver el problema del estancamiento e insuficiencia de los salarios; ${ }^{68}$ por otro lado, se han privatizado y mercantilizado ahorros - otrora públicos - construidos a través de fracciones del salario, posibilitando la financiarización mediante la mercantilización de la esfera reproductiva de la vida social (Fumagalli, 2011; Lapavitsas, 2009a). Ambos elementos dan cuenta del proceso que se ha denominado como expropiación financiera (Lapavitsas, 2013b).

En tercer lugar se produce una recomposición de los grupos económicos que tradicionalmente habían estado anclados en mayor medida en un sector específico de las economías (primario, secundario, terciario), haciéndose predominante la forma holding como táctica de organización de la actividad empresarial, pues permite integrar en una misma unidad económica las actividades de tipo productivo y financiero. Se afirma que tal mecanismo permite una creciente autonomía financiera respecto a los bancos, contribuyendo a construir un entramado de 'finanzas' mucho más extendido que aquel conformado solamente por actores bancarios (Chesnais, 1996; Lapavitsas, 2009a). ${ }^{69}$

\section{El sistema de pensiones chileno: un subsidio colectivo y público a la acumulación empresarial}

Habiendo considerado tales antecedentes se debe destacar que la administración privatizada de los fondos de pensiones está en el corazón de la expansión de las finanzas y de la lógica de financiarización del capital a escala global (Chesnais, 2003; Fumagalli \& Lucarelli, 2011; Lapavitsas, 2009a, 2013b, 2009b). Ante el retiro de la provisión pública de servicios sociales mediante su creciente administración privatizada, diferentes tipos de recursos públicos se han canalizado hacia actores

68 Chile no está excluido de esta tendencia específica de finaciarización de los salarios. Según cifras entregadas por el Banco Central chileno se observa que para el primer trimestre de 2018, el volumen de deuda financiera de los hogares - en relación a sus ingresos anuales - era del 71,5\%; en el último trienio está cifra ha aumentado aproximadamente un 15\% (Banco Central de Chile, 2018).

69 Cada vez más los grupos económicos integran en su seno actividades de tipo financiero: captación de activos financieros, colocación en las bolsas de comercio de acciones o bonos de deuda propios, inserción en el mercado de créditos de consumo, etc. Esto ha sido ampliamente documentado para el caso chileno. Se sugiere revisar los trabajos de Fazio (1997), Caviedes (2015), Caviedes \& Bustamante (2015a, 2015b, 2016a, 2016b) y Barbero (2017). 
privados y desde allí al sistema financiero: la privatización de los fondos de pensiones es uno de los ejemplos más claro de tal tipo de dinámicas (Lapavitsas, 2013a).

En el caso de Chile, es a inicios de la década de 1980 (durante el período dictatorial encabezado por Augusto Pinochet) cuando se configura el momento fundacional del sistema previsional privatizado y por tanto el origen histórico de las administradoras de fondos de pensiones (AFP, de aquí en más). La apertura de este período estuvo marcada por el dictamen del decreto ley número 3.500 en 1981, que dio "inicio al fin de un sistema basado en beneficios definidos, gestionado por múltiples Cajas de Previsión, financiado mediante el mecanismo de reparto, administrado mayoritariamente por el Estado y obligatorio para empleados y obreros en relación de dependencia. Y comenzó a sustituirlo gradualmente por otro de contribuciones definidas, gestionado por Administradoras de Fondos de Pensiones (AFP) reguladas y supervisadas por el Estado, financiado mediante la capitalización en cuentas individuales y obligatorio para los trabajadores en relación de dependencia." (Solimano, 2017, p. 80). Sin entrar a una observación específica sobre la evolución histórica-institucional del sistema previsional chileno, la creciente influencia económica de las AFP en los cursos del capitalismo criollo puede ser detectada atendiendo a la evolución de dos indicadores.

Una primera forma es observar la proporcionalidad entre el tamaño de los fondos previsionales y el volumen del Producto Interno Bruto Nacional (PIB). Según información publicada por la Superintendencia de Pensiones (2018b), entre 1981 y 1985 el crecimiento del fondo de pensiones es sostenido y alcanza a equivaler al 10\% del PIB al final del período indicado. Ya en 1991 supera esa proporción alcanzando casi un tercio del PIB chileno (29\%) y para el año 2000 el volumen de los fondos previsionales representaba la mitad (49\%) del producto nacional anual. Así continuó su crecimiento acelerado durante la primera década del siglo XXI: para el año 2010 ya representaba el 62\% en relación al producto interno bruto, llegando finalmente a una proporción del 72\% en relación al PIB a finales del año 2017.

Este rápido crecimiento de la masa financiera administrada de forma privada va acompañado por una drástica tendencia a la baja en la cantidad de entidades administradoras, lo que se configura como el segundo indicador de la concentración de poder en las AFP chilenas (Superintendencia de Pensiones, 2018b). La tendencia histórica muestra que en un primer momento - aproximadamente en su primera década - la cantidad de administradoras tendió a aumentar. Si en 1981 el sistema partió con 12 AFP, en 1994 existían 21 entidades administradoras. Sin embargo, desde 1995 se inicia una disminución abrupta de la cantidad de administradoras y hacia finales de la década ya se cuentan solamente 8 AFP, estabilizándose la cantidad hacia el año 2004 en alrededor de 6 administradoras, es decir, la misma cantidad que para 2018.

Ahora bien, el papel específico que juegan las AFP en la economía chilena se logra dibujar de forma panorámica al considerar dos evidencias. En primer lugar, se debe considerar que los aportes hechos al sistema previsional en su conjunto (mediante cotizaciones efectuadas por las y los trabajadores y por aportes fiscales directos) llegan a triplicar las pensiones pagadas por las AFP desde los inicios del sistema, considerando el período 1980-2012 (Riesco, Durán, Parelle, \& Lagos, 2012). Así, se comienza a evidenciar que una gran cantidad de dinero ha sido canalizada hacia los administradores del sistema y el mercado financiero. Para contar con una mirada actualizada sobre este asunto se ha compilado información financiera referida al gasto en pensiones del sistema previsional chileno correspondiente tanto al mes de julio de 2018, como al período julio 2017 - julio 2018. La evidencia presentada en la Tabla 1 consigna los gastos, es decir las pensiones pagadas por el sistema previsional chileno diferenciando según su origen (si son pensiones pagadas por el Estado o por el sistema previsional privado) y su destino (es decir, si son pensiones para la población civil o para integrantes de las Fuerzas Armadas y de Orden). ${ }^{70}$

Si se considera sólo el total de gastos efectuados en pensiones civiles se observa que del $100 \%$ del dinero desembolsado por tal concepto, el Estado aporta la mayor parte con un 63\%, mientras que las AFP sólo aportan un $37 \%$ de los recursos gastados. Ahora, si se consideran los gastos totales del sistema previsional chileno, es decir los gastos efectuados tanto por concepto de pensiones de las Fuerzas Armadas y de Orden como para pensiones

70 No se profundizará en aquí sobre tal asunto, pero vale la pena consignar que las Fuerzas Armadas y de Orden en Chile se mantuvieron fuera de la reforma privatizadora de 1980-1981 (Huneeus, 2005), sosteniendo para sí un sistema previsional basado en aportes estatales y asignación de pensiones basada en años de servicio. 
de la población civil, la situación es aún más drástica: se observa que las AFP aportan con sólo un $23 \%$ del total, es decir, escasamente un cuarto del gasto previsional chileno total. En otros términos, esto equivale a decir que el actor que mayor esfuerzo realiza en el pago de pensiones en Chile es el Estado, puesto que asume el $77 \%$ del gasto previsional total.

Tabla 1: Distribución del gasto en pensiones según pilar del sistema previsional chileno (en millones de pesos)

\begin{tabular}{l|c|c|c|c}
\hline \multicolumn{1}{c}{ Ítem/período } & \multicolumn{2}{c}{ Julio 2018} & \multicolumn{2}{c}{ Julio 2017 - julio 2018 } \\
\hline Pensiones civiles & Gasto total & $\%$ gasto total & Gasto total & $\%$ gasto total \\
\hline $\begin{array}{l}\text { Aporte sistema AFP } \\
\text { (pensiones pagadas sin } \\
\text { aportes fiscales) }\end{array}$ & $\$ 188.774$ & $37,1 \%$ & $\$ 2.170 .936$ & $36,1 \%$ \\
\hline $\begin{array}{l}\text { Aporte sistema público } \\
\text { (incluye sistema antiguo, BR } \\
\text { y APS) }\end{array}$ & $\$ 320.193$ & $62,9 \%$ & $\$ 3.841 .448$ & $63,9 \%$ \\
\hline \begin{tabular}{l} 
Total pensiones civiles \\
\hline
\end{tabular} & $\$ 508.957$ & $100,0 \%$ & $\$ 6.012 .383$ & $100,0 \%$ \\
\hline
\end{tabular}

\begin{tabular}{|c|c|c|c|c|}
\hline Pensiones totales & Gasto total & \% gasto total & Gasto total & $\%$ gasto total \\
\hline $\begin{array}{l}\text { Total pensiones civiles } \\
\text { (AFP, PBS, sistema antiguo } \\
\text { reparto) }\end{array}$ & $\$ 508.957$ & $61,5 \%$ & $\$ 6.012 .383$ & $59,6 \%$ \\
\hline Pensiones FFAA y Orden & $\$ 185.117$ & $22,4 \%$ & $\$ 2.292 .112$ & $22,7 \%$ \\
\hline $\begin{array}{l}\text { Otras prestaciones } \\
\text { seguridad social }\left(^{*}\right)\end{array}$ & $\$ 133.570$ & $16,1 \%$ & $\$ 1.776 .905$ & $17,6 \%$ \\
\hline Total sistema previsional & $\$ 827.644$ & $100,0 \%$ & $\$ 10.081 .401$ & $100,0 \%$ \\
\hline Aporte sistema AFP & $\$ 188.774$ & $22,8 \%$ & $\$ 2.170 .936$ & $21,5 \%$ \\
\hline
\end{tabular}

(*) Otras prestaciones: otros subsidios, bonos, reparaciones

\section{Fuente: Elaboración propia con base en CENDA (2018).}

Desde esta perspectiva el actor privado juega un rol bastante minoritario y, se podría decir, poco significativo para los objetivos de un sistema de seguridad social. Aquí se abren una serie de preguntas sobre los elementos que explicarían tal magro aporte del pilar privado: ¿es consecuencia de las deficiencias de cobertura y densidad de cotizaciones o se debe al modo de administración de los recursos en el sistema privado? En términos específicos, ¿Cómo es el flujo de caja de las administradoras privadas?, ¿la mayor parte de los recursos captados por las administradoras vía cotizaciones se destinan al pago de pensiones o se redirigen hacia otro lugar?
Ello nos lleva a la consideración de un segundo indicador sobre el papel de las AFP en la economía nacional, en específico, el flujo de recaudación y gastos del pilar privado del sistema de pensiones en Chile. Considerando el flujo de caja del mes de julio de 2018 se observa que solamente un $32 \%$ del dinero total recaudado (es decir, el flujo de dinero que ingresa al sistema vía descuento salarial o cotizaciones previsionales) se destina a gastos (es decir, al pago de pensiones civiles autofinanciadas). Eso deja con un saldo de $\$ 4,5$ miles de millones de pesos chilenos que son apropiados por el sistema de pensiones privado o, en los términos que tiende a plantearse el debate, resultan 'administrados vía capitalización' en el mercado financiero nacional e internacional. Puede afirmarse que la mayor parte del dinero recaudado por las AFP (un 68\% de las cotizaciones mensuales pagadas desde el actor laboral) no se destinan al pago de pensiones, reteniéndose indefinidamente dentro del circuito financiero del que forman parte las administradoras (CENDA, 2018). Esta afirmación general merece una observación más detallada en relación al papel específico que juegan las administradoras en el entramado del poder económico en Chile, a la vez que respecto a su carácter de clase, anclado en dinámicas financieras.

\section{Conformación de clases sociales en el sistema previsional chileno: 2000-2018}

Una primera cuestión analítica a despejar refiere a cómo delimitar el concepto de clase social. En esta investigación se rescataron tres elementos trabajados por el sociólogo inglés Erik Olin Wright en relación a una definición marxista del concepto de clase, que permitirán establecer dimensiones de análisis para determinar el carácter clasista de las AFP en Chile. Se trata de tres mecanismos relacionales que permiten detectar la existencia de la clase social - como relación y no sólo como categoría - en una situación histórica determinada.

Al respecto Wright indica: i) los intereses materiales, o posición específica de un actor respecto al excedente social; ii) la experiencia histórico-social que orienta una cierta comprensión y visión del mundo, que explica las formas y razones de acción de las colectividades entendidas en un sentido clasista; y iii) las capacidades de acción colectiva, es decir, la capacidad de movilización de recursos para influir en la organización de la sociedad según los intereses materiales y visión particular del 
mundo (Olin Wright, 1992). Si tales características se despliegan de modo sistemático y coherente en el tiempo, para Wright se está en condiciones para poder hablar de la existencia de una clase social. Las siguientes secciones de este apartado proporcionan una caracterización sucinta de las tres dimensiones señaladas. ${ }^{71}$

\section{Las AFP y la acumulación de capital en Chile}

Para delimitar la posición que detentan las administradoras respecto al excedente social, y dilucidar así el papel específico que juegan en las tendencias de la acumulación capitalista en Chile, se observará el carácter de las inversiones efectuadas por las administradoras. Al respecto, la Ilustración 1 muestra la distribución relativa de las inversiones del fondo previsional privado, según el sector institucional de destino para el período 1981 2017. Al respecto, vale la pena destacar tres tendencias.

La primera cuestión relevante es que en el decenio 1986-1996 aumenta de manera sostenida la magnitud de recursos financieros invertidos en el sector "empresas", es decir, en bonos y/o acciones de actores no financieros. Esto va de la mano con una sostenida tendencia a la caída de las inversiones en el sector financiero en el mismo período, elemento que se relaciona, por un lado, con el declive de los grupos financiero-especulativos característicos de la década de 1980 en Chile (Rozas \& Marín, 1988); por otra parte, se vincula con el fuerte papel jugado por las AFP en la formación de una primera base de acumulación financiera para los grupos económicos nacionales que irán ganando mayor musculatura económica ya entrada la primera década de los años 2000 (Barbero, 2017) $^{72}$ y en la inyección de recursos para financiar las diferentes rondas de privatizaciones realizadas por el Estado en Chile (J. P. Arellano, 1985; Huneeus, 2005). ${ }^{73}$

\footnotetext{
71 Para mayores detalles revisar Ruiz (2019).

72 Como se verá, los grupos económicos chilenos de mayor envergadura son quienes han tenido desde las inversiones de los fondos previsionales un claro empuje y rápida disponibilidad de capital para sus proyecciones de crecimiento a escala regional. Una de las razones que explican la presencia de grupos económicos chilenos en elevadas posiciones en los ranking de empresas latinoamericanas (América Economía, 2015), pese a lo reducido de la economía chilena, es precisamente el apalancamiento financiero que vehiculizado por las AFP.

73 Sin entrar en mayores detalles vale la pena señalar que desde finales de la década de 1980 la legislación previsional se modificó para permitir la inversión de los fondos previsionales en empresas que habían sido privatizadas (J. P. Arellano, 1985; Huneeus, 2005).
}

Un segundo aspecto a destacar es que en el período 1997-2002 (post crisis asiática) crecen conjuntamente las inversiones en el sector extranjero y financiero, a la vez que disminuye fuertemente la inversión en el sector estatal; este último aspecto será una tendencia que se proyecta a lo largo de todo el período analizado: si en 1997 la proporción de recursos previsionales invertidos en el sector estatal era del $42 \%$, esta cifra se estabiliza alrededor del 20\% en el período 2011-2017. La tercera cuestión a destacar es que aproximadamente desde 2004 la magnitud de la inversión extranjera es mayor al compararla con cualquiera de los otros sectores: hacia al final del período la inversión extranjera se posiciona levemente por sobre el $40 \%$ de las inversiones totales; si a ello se suman las inversiones nacionales en el sector de las finanzas (aproximadamente 20\%), se observa que del total de fondos de pensiones, más de la mitad (exactamente un 64\%) está invertido en el sector financiero (dado que el 92\% de las inversiones foráneas tienen como destino el sector financiero).

Ilustración 1: Inversión de los fondos de pensiones según sector institucional, en relación al total de activos

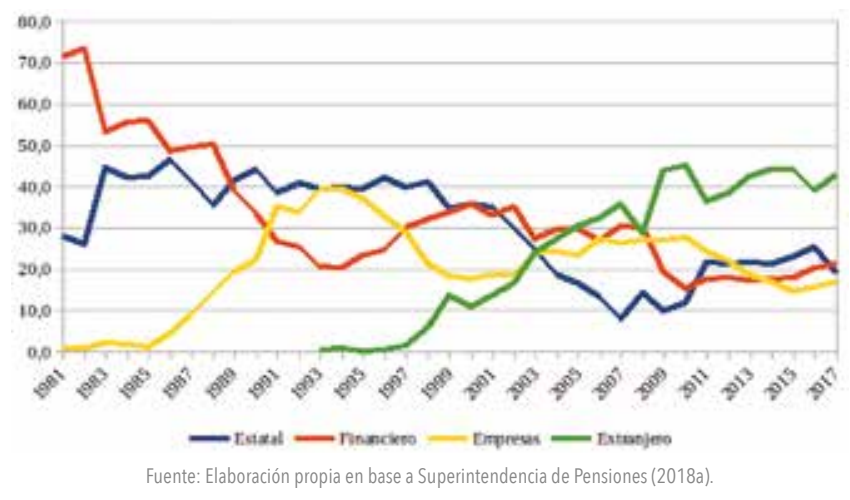

Sobre esta tendencia a la financiarización de los recursos previsionales vale la pena realizar dos observaciones. La primera es que tal tendencia está en línea con la creciente importancia global de las finanzas como mecanismo de acumulación en el capitalismo contemporáneo (Chesnais, 2017; Fumagalli \& Lucarelli, 2011; Lapavitsas, 2013b). La segunda es que, como se verá en el siguiente apartado, tal tendencia está fuertemente relacionada con una progresiva desnacionalización de los recursos previsionales del sistema previsional privado en Chile: por un lado, una parte relevante de los recursos - que, dada la tendencia observada, es posible 
afirmar que seguirá incrementando - se invierte en el extranjero y mayoritariamente en el sector financiero foráneo; por otra, la mayor parte de las administradoras (y principalmente las de mayor tamaño económico) están controladas por conglomerados financieros extranjeros.

Ahora bien, en la actualidad, los actores que hegemonizan la recepción de las inversiones realizadas por las AFP pueden caracterizarse clasificando el destino de tales recursos. $\mathrm{Al}$ analizar una de las variables construida para efectos de esta investigación, que indica la proporción de activos del sistema que son invertidos en grupos económicos, se observa que más de la mitad de los activos del sistema (65\%) se destinan a actores económicos vinculados a importantes grupos económicos nacionales e internacionales. Este elemento refuerza aquella hipótesis planteada tanto por algunos estudios sobre el sistema previsional (Caviedes, 2014; Riesco et al., 2012), como por estudios sobre las características recientes del empresariado en Chile (Barbero, 2017; Dahse, 1979; Rozas \& Marín, 1988; Ruiz \& Boccardo, 2014), que destacan la central importancia que tienen los fondos previsionales como fuente de recursos financieros que permiten dinamizar los ciclos de acumulación y expansión de tales actores.

Para profundizar en la composición interna de las inversiones destinadas a grupos económicos, la Tabla 2 muestra el top 10 de holdings económicos que reciben inversiones desde el sistema de pensiones privado que, en su conjunto, logran captar un poco más de un cuarto $(27 \%)$ del total de las inversiones realizadas por las AFP. Destaca el liderazgo del grupo del empresario chileno Andrónico Luksic en este ranking, que por sí solo logra captar el 4,3\% de la inversión total del sistema de pensiones, siendo la mayor proporción de inversiones destinadas a un grupo económico desde las AFP. De tal modo son 3 grupos económicos de origen estrictamente nacional los que logran posicionarse dentro de este ranking (Luksic, Yarur, Matte); en su conjunto, los tres holding nacionales logran captar el $8,4 \%$ de las inversiones efectuadas por el sistema de pensiones privado en Chile, posicionándose además entre las 10 principales riquezas del país (Forbes, 2017b).

El resto de actores observados (Tabla 2) son importantes compañías a escala mundial (en su totalidad grupos económicos o holdings de tipo financieros). Actores como Blackrock, y Vanguard lideran el mercado de capitales en Norteamérica, mientras que Itaú lo hace en Brasil y América Latina, así como Scotiabank en Europa de forma general, mientras que Santander y BBVA lo hacen en España de forma particular. Todas estas entidades se pueden considerar como compañías de envergadura mayúscula a escala internacional según el ranking Forbes (2017a), a la vez que expresivas de los circuitos de acumulación financiarizada que hoy comandan las rutas de acumulación del capital a escala mundial (Chesnais, 2017; Lapavitsas, 2013b).

Tabla 2: Diez grupos económicos que lideran la captación de recursos desde el sistema de AFP, en porcentajes de la inversión total

\begin{tabular}{cc}
\hline Grupo & Inversión \\
\hline Luksic & $4,30 \%$ \\
Santander & $4,03 \%$ \\
Blackrock & $3,62 \%$ \\
Itaú & $2,97 \%$ \\
Vanguard & $2,93 \%$ \\
Yarur & $2,08 \%$ \\
Matte & $2,06 \%$ \\
Enersis & $1,83 \%$ \\
Scotiabank & $1,71 \%$ \\
BBVA & $1,71 \%$ \\
TOTAL & $\mathbf{2 7 , 2 5 \%}$ \\
\hline
\end{tabular}

Fuente: Elaboración propia en base a (Superintendencia de Pensiones, 2018a).

A partir de la evidencia expuesta puede afirmarse que, en realidad, el sistema de AFP en Chile no constituye “(...) un sistema destinado principalmente al pago de pensiones: es un sistema de ahorro forzoso, un mecanismo de transferencia de una parte de los salarios, hacia los mercados financieros." (Riesco et al., 2012, p. 27) La evidencia mostrada en el presente apartado permite afirmar que el sistema de AFP, más que velar por la seguridad social en la vejez, funciona de manera efectiva como un mecanismo de expropiación financiera, usando el término acuñado por Costas Lapavitsas (2009b, 2013b). En tal sentido, la cotización obligatoria que las y los trabajadores chilenos en situación de dependencia deben obligatoriamente aportar al sistema de AFP, representa una financiarización directa de un $10 \%$ de la masa salarial chilena. Pues como ya se ha visto, considerada como totalidad es destinada en su mayor parte $(64 \%)$ hacia el mercado financiero (ver apartado 2). 


\section{El carácter actual de las administradoras: desnacionalización, concentración y dependencia estatal}

El ordenamiento del mercado previsional en torno seis administradoras permite observar un consolidado marco de actores al finalizar la segunda década de los 2000. En relación al saldo histórico que se observa en la actualidad, vale la pena destacar, en primer lugar, algunos ciclos económicos que marcan diferentes pautas de alianza con capitales internacionales.

La segunda parte de la década de 1990 marca un primer momento de alianza con capitales foráneos, contando como principal antecedente la legalización de diferentes formas de inversión previsional en el extranjero; posteriormente, entre 2000 y 2005 se observa un nuevo re acomodo de alianzas luego de la crisis asiática y antes de la crisis subprime; finalmente, la última crisis mencionada marca un nuevo punto de inflexión, que pavimenta un recambio de los capitales controladores de las administradoras, entrando con fuerza entidades financieras de origen estadounidense: esto determina que actualmente dos de las tres administradoras más grandes (Provida y Cuprum) estén en control total por parte de conglomerados norteamericanos y que la tercera (Habitat) se componga de una alianza entre un grupo empresarial local y capitales estadounidenses. Solamente existe una AFP (Sonda) controlada estrictamente por capitales nacionales y es una de las más pequeñas del mercado. Se observa en tal sentido - como ya ha sido señalado - una fuerte tendencia a la financiarización de las inversiones previsionales, en conjunto con una intensa desnacionalización de los fondos de pensiones: se trataría de un aterrizaje de intereses financieros internacionales, férreamente asentados en la economía chilena mediante las administradoras de fondos previsionales, que desde allí establecen pautas de alianza con grandes capitales locales e internacionales.

Así, en segundo término, se observa que el control de los fondos previsionales está fuertemente concentrado en un reducido número de actores. Las tres AFP de mayor envergadura - Habitat, Provida y Cuprum - concentran el $72 \%$ de los fondos previsionales y el $54 \%$ de afiliados/ as que cotizan. Adicionalmente, tales administradoras de mayor envergadura también son aquellas donde las finanzas internacionales se han posicionado con mayor fuerza, mostrando cómo la trayectoria de desnacionalización también se asocia a una fuerte tendencia a la concentración del control de los fondos previsionales de las y los trabajadores en Chile. La propiedad de la AFP Habitat se reparte equitativamente entre Prudential Finantial Group (Forbes, 2018b) y la Cámara Chilena de la Construcción, ${ }^{74}$ la propiedad de Provida está en manos del conglomerado Metlife (Forbes, 2018a), mientras que el control de Capital está en manos del conglomerado colombiano Sura Asset Management (Reuters, 2011).

Un tercer y último aspecto a destacar de esta ruta de transformaciones históricas es la fuerte dependencia de la acción estatal en las pautas de acción de las administradoras. Por una parte, todo su arsenal de inversiones se ha desarrollado por decreto: ha dependido de la continua modificación de las regulaciones asociadas a la cartera de inversiones de los fondos previsionales, que desde sus inicios ha ido relajando las normativas en relación a los tipos y magnitudes de inversión financiera que podían ser realizados por estas entidades. Por otra parte, también destaca que la misma aparición de nuevos actores en el mercado previsional ha estado determinada por la acción del Estado. Desde la Reforma Previsional de 2008, en la cual se definió un mecanismo de licitación para la de adjudicación de la cartera de nuevos afiliados al sistema, se configuró un soporte específico para el desarrollo de actores nuevos en el mercado de las pensiones y para aquellas administradoras de menor tamaño, lo que ha permitido que sigan existiendo posiciones minoritarias del mercado previsional administradas por capitales locales.

La reforma señalada posibilitó la creación de la AFP Sonda, que se adjudicó los dos primeros ciclos de licitación $(2010,2012)$. Posteriormente la otra AFP de menor tamaño del sistema previsional, PlanVital, orientó su estrategia de mercado hacia la captura de tal mercado cautivo, ganando las dos licitaciones siguientes (2014, 2016). Actualmente, ante la posibilidad de que se reactive tal mecanismo de licitación ya se ha creado una nueva AFP de nombre UNO (EMOL, 2018), cuya

74 La trayectoria reciente de la Cámara Chilena de la Construcción es expresiva de la consolidación de un capitalismo de servicios públicos y financiero en la modernización neoliberal chilena. Para más detalles sobre el papel de este conglomerado en la administración de AFP Habitat ver Ruiz Bruzzone (2019); para un estudio en profundidad sobre su evolución histórica ver el trabajo en desarrollo de Kornbluth (2019). 
propiedad es de antiguos gerentes de la histórica AFP Cuprum, que de tal forma esperan tener una rápida inserción en el mercado previsional.

\section{Las AFP como actor colectivo: de la pax previsional al conflicto social (1990-2018)}

En relación a las dinámicas de acción colectiva de las administradoras vale la pena destacar su capacidad de agencia hacia el poder político y la acción estatal. Resulta interesante notar que tanto durante la década de 1990 como durante la primera década de los 2000, en el debate público no lograron tener incidencia posturas críticas hacia la estructuración del sistema previsional en Chile (Arenas, 2010). Como es señalado por BrilMascarenhas y Maillet (2018), durante todo el período democrático se observa una intensa "puerta giratoria" entre la industria previsional y altos puestos de la administración del Estado. Tal política se tradujo en un sostenido reclutamiento de figuras políticas clave para directorios y juntas de accionistas de las administradoras, revelando un esfuerzo sistemático por parte de las AFP para construir un anillo de tomadores de decisiones políticas "amigables" con sus intereses, lo que les brindó una elevada capacidad de respuesta e intervención directa sobre las decisiones de política pública, logrando clausurar el debate público en torno a cualquier posible reforma del sistema de pensiones en Chile que alterara su lógica basal (la financiarización de los salarios) por casi tres décadas (Ruiz Bruzzone, 2019).

En relación al mundo del trabajo, durante la década de 1990 y los primeros doce años del siglo XXI se observó un escenario en el que de manera muy aislada comenzaron a emerger tímidas expresiones de crítica y contra propuestas al sistema previsional en curso (Mejías \& Panes, 2018). En el año 2000 La Asociación Nacional de Empleados Fiscales (ANEF) desarrolló una propuesta de reparación para el daño previsional que significó el paso forzoso al sistema de AFP para las y los trabajadores del Estado, apuntando a un retorno paulatino a la lógica de las antiguas cajas de previsión de carácter sectorial, sin lograr su implementación. Por otra parte, en una serie de movilizaciones ocurridas entre 2002 y 2006 los sindicatos agrupados en la Unión Portuaria de Chile (UPCH) también propusieron la estructuración de un sistema de reparto financiado de forma tripartita sin ganar tal demanda. De forma posterior a la crisis subprime de
2008, la Confederación de Sindicatos Bancarios y Afines organizaron una serie de acciones legales contra las AFP para reparar el daño previsional que significó la pérdida de recursos producto de la crisis subprime, que poco a poco fueron instalando la idea del aprovechamiento financiero de tales recursos. Finalmente, para el año 2012 se comenzó a constituir en la zona de Concepción el Movimiento Sindical por la Seguridad Social y la Previsión, que hizo confluir al sindicalismo del sector portuario, organizaciones estudiantiles y trabajadores del sector salud en un antecedente de lo que un años después (2013) se constituyó como la Coordinadora NO+AFP (Rozas Bugueño \& Maillet, 2019).

Se observa, entonces, un punto de quiebre en la dinámica histórica anotada, pues se comienza a conformar una polaridad clasista en torno al sistema previsional chileno. A partir de la creación en Junio de 2013 de la Coordinadora Nacional de Trabajadores y Trabajadoras $\mathrm{NO}+\mathrm{AFP}$ gracias a la confluencia de organizaciones sindicales diversas, por fuera de los sectores sindicales tradicionales en Chile, emerge un actor sindical cuya organización y horizonte de lucha tiene que ver con un aspecto que escapa a la laboral en sentido estricto, pues se orienta a construir mejores condiciones para la reproducción de la vida una vez terminada la edad económicamente activa (Mejías \& Panes, 2018), en una abierta confrontación frente al empresariado del sistema de pensiones (Rozas Bugueño \& Maillet, 2019).

En paralelo, comenzó un proceso de deslegitimación del sistema previsional alimentado por dos cauces. El primero fueron los diagnósticos entregados por la Comisión Asesora Presidencial para la Reforma del Sistema de Pensiones construida en el segundo gobierno de Michelle Bachelet (Comisión Presidencial Pensiones, 2015): de modo inédito, esta comisión de expertos mostró que pese a su magnitud económica y gigantes rentabilidades privadas, el sistema de pensiones se mostraba insuficiente para entregar pensiones en la vejez y que requería ajustes sustantivos (Bril-Mascarenhas \& Maillet, 2018). En segundo término, durante 2015 y 2016 salieron a la luz una serie de casos de pensiones aumentadas arbitrariamente en las fuerzas armadas, suceso que provocó un creciente descontento popular en torno al tema previsional (González \& Aristeo, 2016) al evidenciarse cómo este grupo de poder accedía a pensiones desorbitantemente mejores que aquellas recibidas por la 
población civil; durante el mismo año, también salieron a la luz pública los "perdonazos" tributarios concedidos a Principal Financial Group y Metlife Financial Group para la consolidación de sus negocios previsionales en Chile mediante una exención tributaria por casi US $\$ 400$ millones en total (Cárcamo, 2017).

Así, durante 2016 se observaron diferentes iniciativas de acción por parte de la Coordinadora NO+AFP que escapan a la lógica de acción sindical remitida al ámbito de la empresa (Mejías \& Panes, 2018; Rozas Bugueño \& Maillet, 2019). En primer lugar puede señalarse la campaña por que las y los trabajadores se cambiaran al fondo previsional de menor riesgo con el objetivo de proteger los ahorros previsionales de los impactos negativos de las fluctuaciones financieras. En segundo término, también destacó el llamado a abandonar las AFP Cuprum y Provida efectuado por la Coordinadora luego de conocerse públicamente los beneficios obtenidos por tales administradoras en los perdonazos tributarios ya mencionados. En tercer lugar, durante 2016, la coordinadora presentó públicamente una propuesta de sistema previsional alternativo, basado en una lógica de solidaridad intergeneracional, con aportes tripartitos y un mecanismo de reparto para la asignación de pensiones (No+AFP, 2016); durante 2017 esta propuesta se validó en un plebiscito ciudadano organizado por la coordinadora sindical, en el cual participaron un millón de personas que aprobaron la propuesta con una mayoría cercana al 99\% (Rozas Bugueño \& Maillet, 2019).

Estas pautas de acción se configuran como otro indicador de la existencia de un proceso de conformación de clases sociales al alero del sistema previsional chileno, que resulta expresiva de las nuevas formas de configuración de empresariado en Chile. Lo que se configura desde el mundo del trabajo es un actor colectivo que, en base a una interés subalterno sobre el excedente social, configura una experiencia y visión compartida sobre la sociedad, y se organiza colectivamente a partir de una polaridad de oposición al empresariado del sistema previsional, pretendiendo incidir en la estructuración de la vida colectiva. Esta dinámica, más allá de los resultados específicos en la arena política que haya logrado el movimiento (la efectiva transformación o impugnación del sistema previsional privado, que en efecto, no ha ocurrido) revela una lógica clasista en la estructuración de estos actores colectivos (Olin Wright, 1992).

\section{Nuevas formas de acumulación capita- lista y desafíos contemporáneos para la democracia}

En las líneas anteriores se ha buscado mostrar la profunda afinidad existente entre la financiarización de las economías, como una característica novedosa del capitalismo neoliberal, y el proceso reciente de conformación de clases sociales en Chile (Ruiz Bruzzone, 2019). Utilizando los aportes ya clásicos del marxismo analítico (Olin Wright, 1992) se ha descrito la conformación de un actor colectivo en torno al sistema previsional chileno, las administradoras de fondos previsionales, que en el curso de la historia reciente se han ido constituyendo como una fracción empresarial con marcados ribetes clasistas.

El empresariado previsional es un actor novedoso en términos de la posición que ocupa en los circuitos de acumulación: se ubica por fuera de la relación salarial, en una dinámica de expropiación financiera (Lapavitsas, 2009b) que le permite articular diferentes capas del empresariado nacional e internacional, posibilitando tanto la entrada como salida de capitales, en relación a la economía nacional, sirviendo de "espalda financiera" para la expansión dependiente pero asociada del empresariado local (Cardoso \& Faletto, 1977). Asimismo, su carácter financiarizado y desnacionalizado le otorga una ubicuidad propia de los circuitos de acumulación contemporáneos (Chesnais, 2017). La influencia que ha logrado en relación al poder político le brinda una amplia gama de posibilidades de acción colectiva, aunque en el último tiempo ha debido enfrentar la constitución de una polaridad social antagónica pues desde la clase trabajadora se ha comenzado a articular un actor que pretende disputar su posición e intereses en la sociedad chilena (Bril-Mascarenhas \& Maillet, 2018; Rozas Bugueño \& Maillet, 2019).

En un momento en que la égida progresista parece venir en retroceso en América Latina, sin haber logrado consolidar un modelo de desarrollo alternativo al capitalismo neoliberal (Ferretti \& Ruiz Bruzzone, 2018), la observación de estas dinámicas permite apuntar las principales taras del carácter político y social de la democracia en Chile. Proponemos que tales cuestiones apuntan a los desafíos de mayor envergadura para las fuerzas de izquierda y progresistas no sólo en este país, 
pues resultan ser condiciones homologables a una amplia diversidad de sociedades en latinoamérica. Por el lado de su configuración política, destaca como desafío táctico la posibilidad de superar el efectivo blindaje ejercido por el denominado "pacto de la transición" en Chile, en el cual la democratización política se subordinó al cierre social del Estado en torno a una estrecha alianza entre cúpulas políticas y la clase económicamente dominante. Por el lado social, la superación de la creciente distancia entre sociedad y política es un importante desafío estratégico: la constricción de la democracia así como la neoliberalización de la política se han apoyado tanto en la masiva desarticulación de la organización colectiva en el campo laboral (Ruiz Bruzzone, 2016), como en el vaciamiento social de la política democrática representativa (F. Arellano, Plaza, \& Ruiz, 2017),

El sustrato de la llegada al poder de presidentes de signo derechista en la región es el avance de las fuerzas del capitalismo sobre la democracia y la política. En un momento donde el ciclo de los commodities viene en bajada, la puja por ampliar las esferas susceptibles de mercantilización será vital para que el ciclo largo iniciado con el giro neoliberal de las décadas de 1980-1990 siga teniendo vida. Desde esta perspectiva, el proceso examinado para el caso chileno indica la necesaria centralidad de una ampliación del carácter social de la política y una radicalización de las posibilidades de dirimir democráticamente el curso histórico de nuestras sociedades. Es la tensión mercado-democracia aquella que hoy en día otorga dinamismo a las posibilidades de construcción de una polaridad social que amplíe los marcos de ordenamiento de nuestras colectividades, más allá de las constricciones del capital y el mercado, superando el techo burocrático-estatal de aquellos gobiernos autodenominados progresistas (Ruiz, 2019) pero que, en el ciclo largo, no construyeron una fuerza social alternativa al neoliberalismo.

\section{Referencias}

América Economía. (2015). Ranking: Las 500 mayores empresas de Chile. Revista América Economía, 50-69.

Arellano, F., Plaza, A., \& Ruiz, F. (2017). Más allá del cascarón vacío: el carácter social de la abstención electoral en el Chile del siglo XXI. Revista Cuadernos de Coyuntura, (20), 3-8.

Arellano, J. P. (1985). Capítulo III. La reforma previsional chilena tres años después. En Políticas Sociales y Desarrollo. Chile 1924-1980, 137-223. Recuperado de http://www.cieplan.org/biblioteca/detalle.tpl?id=41
Arenas, A. (2010). Historia de la reforma previsional chilena: una experiencia exitosa de política pública en democracia. Santiago de Chile: Oficina Internacional del Trabajo.

Banco Central de Chile. (2018). Cuentas Nacionales. Recuperado de Base de Datos Estadísticos website: https://si3.bcentral.cl/Siete/secure/cuadros/home. aspx

Barbero, M. I. (2017). Las multinacionales chilenas: contextos, trayectorias, estrategias. En Empresas y empresarios en la historia de Chile: 1930-2015. Santiago de Chile: Editorial Universitaria, 235-284.

Bril-Mascarenhas, T., \& Maillet, A. (2018). How to Build and Wield Business Power: The Political Economy of Pension Regulation in Chile, 1990-2018. Latin American Politics and Society, 60(4), 1-25. https://doi.org/10.1017/lap.2018.61

Cárcamo, H. (2017). 2016, el año en que la industria de AFP tocó fondo y comenzó a vivir en peligro. El Mostrador. Recuperado de https://www. elmostrador.cl/mercados/2017/01/30/2016-el-ano-en-que-la-industria-de-afptoco-fondo-y-comenzo-a-vivir-en-peligro/

Cardoso, F. H., \& Faletto, E. (1977). Post scriptum a «Dependencia y desarrollo en América Latina». Desarrollo Económico, 17(66), 273-299. https:// doi.org/10.2307/3466399

Caviedes, S. (2014). Los trabajadores como pilar del sistema financiero. El botín detrás de las AFP. Cuadernos de Coyuntura, 6, 38-49.

Caviedes, S. (2015). Cuando un crédito por $\$ 6.500$ millones poco importa: la verdadera influencia empresarial del grupo Luksic. Cuadernos de Coyuntura, 7, 37-50.

Caviedes, S., \& Bustamante, A. (2015a). El papel de la tradición: la influencia empresarial del grupo Matte. Cuadernos de Coyuntura, 8, 40-54.

Caviedes, S., \& Bustamante, A. (2015b). La red de arrastre estatal: la influencia empresarial del grupo Angelini. 9, 1-12.

Caviedes, S., \& Bustamante, A. (2016a). Endeudar para acumular: la influencia empresarial del grupo Solari. Cuadernos de Coyuntura, 13.

Caviedes, S., \& Bustamante, A. (2016b). La precariedad laboral como fórmula de emprendimiento: la influencia empresarial del grupo Paulmann. Cuadernos de Coyuntura, 12, 32-44.

CENDA. (2018). IMACEI - Índices Mensuales de Actividad Económica Interna. Recuperado 29 de septiembre de 2018, de Centro de Estudios Nacionales de Desarrollo Alternativo website: https://www.cendachile.cl/seriescenda/indice-mensual-de-actividad-economica-interna-imacei---cenda

Chesnais, F. (1996). La «globalización» y el estado del capitalismo a finales de siglo. Investigación Económica, 56(215), 233-269.

Chesnais, F. (2003). La teoría del régimen de acumulación financiarizado: contenido, alcance e interrogantes. Revista de Economía Crítica, 1, 37-72.

Chesnais, F. (2017). Finance capital today. Corporations and banks in the Lasting Global Slump. Chicago: Haymarket Books.

Comisión Presidencial Pensiones. (2015). Informe Final. Comisión Asesora Presidencial sobre el Sistema de Pensiones. Recuperado de Comisión Asesora Presidencial Sobre el Sistema de Pensiones website: www.comision-pensiones. cl/Informe_final_CP_2015.pdf

Dahse, F. (1979). El mapa de la extrema riqueza: los grupos económicos y el proceso de concentración de capitales. Santiago: Editorial Aconcagua.

Duménil, G., \& Lévy, D. (2001). Costs and benefits of neoliberalism. A class analysis. Review of International Political Economy, 8(4), 578-607. https://doi. org/10.1080/09692290110077593

EMOL. (2018). UNO AFP, la gestora del ex Cuprum Ignacio Álvarez que espera la licitación para entrar a competir por los nuevos afiliado. El Mercurio Online. Recuperado de http://www.economiaynegocios.cl/noticias/noticias. asp? $\mathrm{id}=506866$ 
Encuentros / Formación de clases y conflicto social en el sistema previsional chileno: mecanismos de acumulación capitalista...

Escalante, F. (2016). Historia mínima del neoliberalismo. México: El Colegio de México.

Fazio, H. (1997). Mapa actual de la extrema riqueza en Chile. Santiago de Chile: LOM Ediciones.

Ferretti, P., \& Ruiz Bruzzone, F. (2018). Ampliación de la política y desmercantilización de la vida social. Claves para proyectar la superación del neoliberalismo en Chil. Revista Trama, 4(Posneoliberalismo: estrategias y perspectivas), 13-19.

Forbes. (2017a). The World's Biggest Public Companies. Ranking 2017. Recuperado de Forbes Magazine website: https://www.forbes.com/global2000/ list/\#tab:overall

Forbes. (2017b). The World's Billionaire List. Ranking 2017. Recuperado de Forbes Magazine website: https://www.forbes.com/billionaires/ list/\#version:static_country:Chile

Forbes. (2018a). MetLife on the Forbes Global 2000 List. Recuperado 20 de noviembre de 2018, de Forbes website: https://www.forbes.com/companies/metl ife/?list=global2000\#39aef52c4aef

Forbes. (2018b). Prudential Financial on the Forbes Global 2000 List. Recuperado 20 de noviembre de 2018, de Prudential Finantial website: https:// www.forbes.com/companies/prudential-financial/

Fumagalli, A. (2011). Twenty Thesis on Contemporary Capitalism (cognitive biocapitalism). Angelaki: Journal of the Theoretical Humanities, 16(3), 7-17. https://www.tandfonline.com/doi/abs/10.1080/0969725X.2011.626555

Fumagalli, A., \& Lucarelli, S. (2011). A financialized monetary economy of production. international journal of political economy. 40(1), 48-68.

González, V., \& Aristeo, A. (2016). "Jubilazos" en Gendarmería: CDE se querella por fraude al Fisco e individualiza caso de Olate. Bio Bio Chile. Recuperado de https:/www.biobiochile.cl/noticias/nacional/chile/2016/10/07/ jubilazos-en-gendarmeria-cde-se-querella-por-fraude-al-fisco-e-individualizacaso-de-olate.shtml

Huneeus, C. (2005). El régimen de Pinochet. Editorial Sudamericana.

Kornbluth, D. (2019). Neoliberalización y empresariado de la construcción en Chile (1951-2015) (Tesis doctoral en desarrollo, Universidad de Chile). Recuperado de https://www.coes.cl/integrantes/david-kornbluth/

Lapavitsas, C. (2009a). Financialisation, or the search for profits in the sphere of circulation. EKONOMIAZ. Revista Vasca de Economía, 72(03), 98-119.

Lapavitsas, C. (2009b). Financialised Capitalism: Crisis and Financial Expropriation. Historical Materialism, 17(2), 114-148.

Lapavitsas, C. (2013a). Financialisation and capitalist accumulation: A structural account of the crisis of 2007-9. En K. Yagi, N. Yokokawa, S. Hagiwara, \& G. Dymski (Eds.), Crises of Global Economies and the Future of Capitalism, 5574. Recuperado de https://www.routledge.com/Crises-of-Global-Economy-andthe-Future-of-Capitalism-An-Insight-into/Yagi-Yokokawa-Shinjiro-Dymski/p/ book/9780415687331

Lapavitsas, C. (2013b). The financialization of capitalism: 'Profiting without producing'. City, 17(6), 792-805. https://doi.org/10.1080/13604813.2013.8538 65

Lapavitsas, C. (2016). Beneficios sin producción. Cómo nos explotan las financas. Recuperado de https://www.traficantes.net/libros/beneficios-sinproducci\% $\% 3 \% \mathrm{~B} 3 \mathrm{n}$

Mejías, S., \& Panes, D. (2018). Reestucturación neoliberal y crisis del sistema previsional. Análisis a partir de las transformaciones del Estado y la acción de los trabajadores en Chile. En: Desigualdades, exclusión y crisis de sustentabilidad en los sistemas previsionales de América Latina y el Caribe, Buenos Aires: CLACSO, 47-83. Recuperado de http://www.clacso.org.ar/libreria-latinoamericana/libro_ detalle.php?id_libro=1431
No+AFP. (2016). Nuevo Sistema de Pensiones para Chile. Recuperado de Coordinadora Nacional de Trabajadores NO+AFP website: http:// coordinadoranomasafp.cl/wp/un-nuevo-sistema-previsional-para-chile-pdf/

O'Donnell, G. (1977). Reflexiones sobre las tendencias de cambio del Estado burocrático-autoritario. Revista mexicana de sociología, 9-59.

Olin Wright, E. (1992). Reflexionando, una vez más, sobre el concepto de estructura de clases. Zona abierta, (59), 17-126.

Reuters. (2011). Grupo colombiano Sura adquirirá activos de ING en Latinoamérica en US\$3.763 millones.

Riesco, M., Durán, F., Parelle, A., \& Lagos, F. (2012). Resultados para sus afiliados de las AFP y Compañías de Seguros relacionadas con la previsión 1982 2012. Santiago de Chile: CENDA.

Rozas Bugueño, J., \& Maillet, A. (2019). Entre marchas, plebiscitos e iniciativas de ley: innovación en el repertorio de estrategias del movimiento No Más AFP en Chile (2014-2018). Izquierdas, 48, 1-21.

Rozas, P., \& Marín, G. (1988). El 'Mapa de la Extrema Riqueza' diez años después. Santiago de Chile: Ediciones Chile América - CESOC.

Ruiz Bruzzone, F. (2016). Panorámica del Trabajo en el Chile Neoliberal. Santiago de Chile: Editorial Universitaria

Ruiz Bruzzone, F. (2019). El empresariado del sistema de pensiones en Chile (2000-2018) (Tesis para optar al grado de magíster en Ciencias Sociales, con mención en Sociología de la Modernización y al título profesional de sociólogo). Universidad de Chile, Santiago de Chile

Ruiz, C. (2019). La política en el neoliberalismo. Experiencias latinoamericanas. Santiago de Chile: LOM Ediciones.

Ruiz, C., \& Boccardo, G. (2014). Los chilenos bajo el neoliberalismo. Clases y conflicto social. Santiago de Chile: El Desconcierto-Fundación Nodo XXI.

Solimano, A. (2017). Pensiones a la chilena. La experiencia internacional y el camino a la desprivatización. Santiago de Chile: Catalonia.

Superintendencia de Pensiones. (2018a). Estadísticas e informes. Recuperado 15 de septiembre de 2018, de Superintendencia de Pensiones website: https:// www.spensiones.cl/portal/institucional/594/w3-propertyname-621.html

Superintendencia de Pensiones. (2018b). Series estadísticas del sistema de pensiones. Recuperado de Superintendencia de Pensiones website: http://www. spensiones.cl//safpstats/stats/.sc.php?_cid=41

Torres, E. (2017). El proyecto intelectual: hacia la reconstrucción de un programa teórico para las ciencias sociales en América Latina. Cuadernos del pensamiento crítico latinoamericano, 48, 1-4. 